\title{
Association between F-18 fluorodeoxyglucose uptake of noncancerous lung area and acute exacerbation of interstitial pneumonia in patients with lung cancer after resection
}

Takashi Yamamichi, MD, ${ }^{\mathrm{a}}$ Yoshihisa Shimada, MD, PhD, ${ }^{\mathrm{a}}$ Ryuhei Masuno, MD, PhD, ${ }^{\mathrm{b}}$ Tatsuo Ohira, MD, PhD, ${ }^{a}$ Shinji Abe, MD, PhD, ${ }^{\mathrm{c}}$ Akinobu Yoshimura, MD, PhD, ${ }^{\mathrm{d}}$ and Norihiko Ikeda, $\mathrm{MD}, \mathrm{PhD}^{\mathrm{a}}$

\section{ABSTRACT}

Background: Idiopathic pulmonary fibrosis is defined as a specific form of progressive fibrosing interstitial pneumonia. Postoperative acute exacerbation is considered a lethal comorbidity for patients with lung cancer, particularly when it is accompanied with idiopathic pulmonary fibrosis. Thus, pretherapeutic risk stratification for acute exacerbation has been anticipated. In this study, we aimed to investigate whether the maximum standardized uptake value of F-18 fluorodeoxyglucose is useful for assessing the postoperative risk of acute exacerbation and severe respiratory adverse events in patients with lung cancer after surgical resection.

Methods: A total of 822 patients with lung cancer who underwent preoperative high-resolution computed tomography, fluorodeoxyglucose-positron emission tomography/computed tomography, and pulmonary resection between July 2012 and July 2018 were assessed. Maximum standardized uptake value of the main tumor and that of the noncancerous lung area were measured using a 3-dimensional workstation. Multivariable analyses for acute exacerbation and severe respiratory adverse events were performed using the logistic regression model.

Results: Among all patients, 120 (14.6\%) had idiopathic pulmonary fibrosis findings on high-resolution computed tomography whereas severe respiratory adverse events were observed in $35(4.2 \%)$ patients, including those with acute exacerbation $(\mathrm{n}=15,1.8 \%)$. Maximum standardized uptake value of the main tumor and that of the noncancerous lung area were independently associated with both acute exacerbation and severe respiratory adverse events on multivariable analysis, both in all patients and in the 120 patients with idiopathic pulmonary fibrosis. Risk stratification analysis showed that $19.0 \%$ and $30.2 \%$ of patients who were positive for idiopathic pulmonary fibrosis on high-resolution computed tomography and with a maximum standardized uptake value of the main tumor and that of the noncancerous lung area 1.69 or greater (the optimal cutoff value relevant to acute exacerbation) experienced acute exacerbation and severe respiratory adverse events, respectively.

Conclusions: Maximum standardized uptake value of the main tumor and that of the noncancerous lung area were independently associated with the incidence of postoperative acute exacerbation and severe respiratory adverse events in patients with lung cancer. (J Thorac Cardiovasc Surg 2020;159:1111-8)

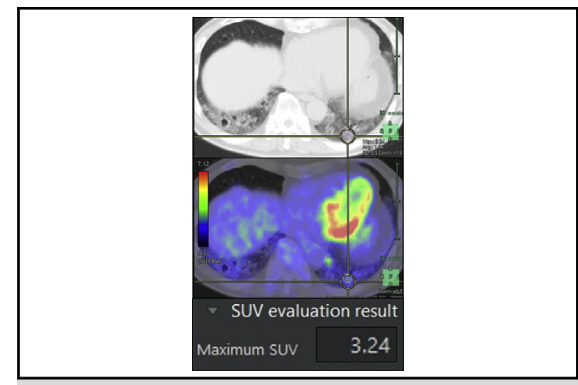

Measurement of the SUVmax of noncancerous lung area using a 3-dimensional workstation.

\section{Central Message}

Maximum standardized uptake value of noncancerous lung area is significantly associated with the incidence of $\mathrm{AE}$ of interstitial pneumonia in patients after lung cancer surgery.

\section{Perspective}

This study aims to investigate whether SUVmax of F-18 FDG can be used to assess the risk of postoperative $\mathrm{AE}$ of IP in patients with lung cancer after surgical resection. Clinicosurgical variables, including metabolic metrics on FDG-PET, are expected to be useful for the determination of appropriate treatment strategies for patients with lung cancer with IP.

See Commentary on page 1119.

\footnotetext{
From the Departments of ${ }^{\mathrm{a}}$ Surgery, ${ }^{\mathrm{b}}$ Radiology, ${ }^{\mathrm{c}}$ Respiratory Medicine, and ${ }^{\mathrm{d}}$ Medical Oncology, Tokyo Medical University, Tokyo, Japan.

Received for publication April 3, 2019; revisions received July 24, 2019; accepted for publication July 25, 2019; available ahead of print Oct 3, 2019.
}

\footnotetext{
Address for reprints: Yoshihisa Shimada, MD, PhD, Department of Surgery, Tokyo Medical University, 6-7-1, Nishishinjuku, Shinjuku-ku, Tokyo, 160-0023, Japan (E-mail: zenkyu@za3.so-net.ne.jp).

$0022-5223 / \$ 36.00$

Copyright (C) 2019 by The American Association for Thoracic Surgery

https://doi.org/10.1016/j.jtcvs.2019.07.100
} 


$$
\begin{aligned}
& \text { Abbreviations and Acronyms } \\
& \begin{aligned}
\text { AE } & =\text { acute exacerbation } \\
\text { CT } & =\text { computed tomography } \\
\text { FDG } & =\text { fluorodeoxyglucose } \\
\text { IP } & =\text { interstitial pneumonia } \\
\text { IPF } & =\text { idiopathic pulmonary fibrosis } \\
\text { NCA } & =\text { noncancerous lung area } \\
\text { PET } & =\text { positron emission tomography } \\
\text { ROC } & =\text { receiver operating characteristic } \\
\text { SRAE } & =\text { severe respiratory adverse event } \\
\text { SUVmax } & =\text { maximum standardized uptake value } \\
\text { UIP } & =\text { usual interstitial pneumonitis } \\
\% \mathrm{VC} & =\text { percentage vital capacity }
\end{aligned}
\end{aligned}
$$

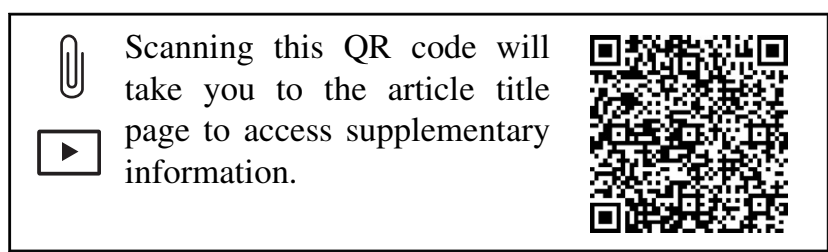

The incidence of lung cancer in patients with interstitial pneumonia (IP), most of which is idiopathic pulmonary fibrosis (IPF), is higher than in the general population, and relative risks were reported to be between $7 \%$ and $14 \% .{ }^{1}$ In patients with IPF with lung cancer, postoperative acute exacerbation (AE) is considered a lethal comorbidity with a high mortality rate of $30 \%$ to $100 \%{ }^{1,2}$ A recent report of a multicenter cohort study in Japan demonstrated that postoperative AE occurred in $0.5 \%$ of all surgical cases of lung cancer. ${ }^{3}$ In addition, the prevalence of IPF has been found to increase considerably with age. ${ }^{4}$ Therefore, it is important to assess the postoperative risk of $\mathrm{AE}$; however, there is little consensus about which criteria are the most appropriate to identify patients at high risk of AE after surgical resection for lung cancer.

Recent studies identified a metabolic shift in patients with IPF, with increased glycolysis, particularly in their fibroblasts. $^{5-7}$ The cellular mechanism underlying increased F-18 fluorodeoxyglucose (FDG) accumulation in malignant tumors is associated with a higher rate of glucose transport across the cell membrane. ${ }^{8}$ FDG- positron emission tomography (FDG-PET) contributes to the staging, qualitative diagnosis, and assessment of the therapeutic effects of treatments for lung cancer management. ${ }^{9,10}$ FDG-PET signals were also found to be consistently increased and measurable in some patients with IPF. ${ }^{11}$ PET signals were increased in the area of honeycombing, in which these fibrosis regions may be active. ${ }^{12}$ Nobashi and colleagues ${ }^{11}$ showed that increased F-18 FDG uptake in the lung parenchyma was associated with poor survival in patients with IP. Flechsig and colleagues ${ }^{13}$ also showed that F-18 FDG-PET/computed tomography (CT) was able to detect patients at high risk for progressive IPs. These findings suggest that F-18 FDG uptake is likely to be associated with the severity of the fibrotic process, and there are common molecular mechanisms that causes DNA damage in both cancer and IPF. ${ }^{14,15}$ We speculated that the incidence of postoperative $\mathrm{AE}$ may be relevant to preoperative FDG-PET signals in the noncancerous lung areas of patients with lung cancer, particularly in those with accompanying IPF. Therefore, in this study we aimed to investigate whether FDG-PET/CT can be used to assess the risk of postoperative $\mathrm{AE}$ and severe respiratory adverse events (SRAEs) in patients who underwent surgical resection for primary lung cancer (Video 1 and Figure 1).

\section{PATIENTS AND METHODS \\ Patients}

We identified 822 patients with lung cancer who underwent preoperative FDG-PET/CT in a designated facility for this study and underwent subsequent pulmonary resection between July 2012 and July 2018 (Figure 2). We reviewed the patients' clinical records, including age, sex, smoking history, CT and FDG-PET/CT imaging findings, results of pulmonary function tests, surgical records, and postoperative adverse events. Postoperative adverse events were evaluated on the basis of the Clavian-Dindo classification. ${ }^{16}$ IPF was diagnosed in accordance with the American Thoracic Society/European Respiratory Society/The Japanese Respiratory Society/Latin American Thoracic Association IPF criteria on high-resolution CT (HRCT). ${ }^{17}$ Radiologic findings (1: usual interstitial pneumonitis [UIP]; 2: probable UIP; 3: non-UIP, including

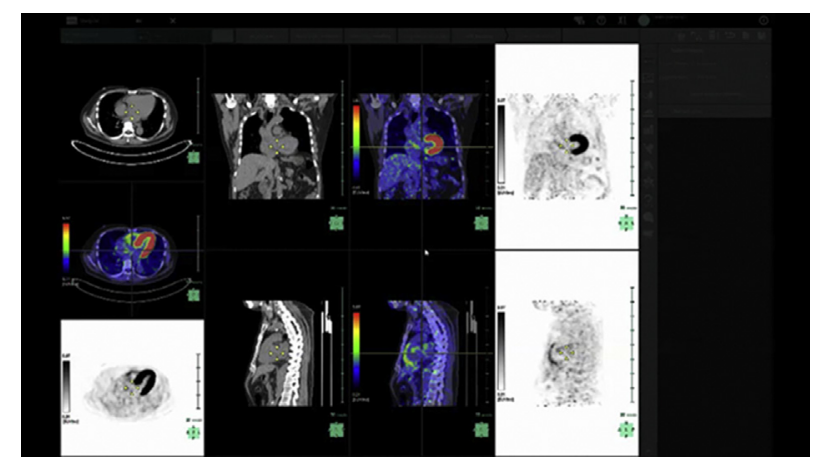

VIDEO 1. Flow chart of the current study for the purpose of identifying factors associated with acute exacerbation (AE) of interstitial pneumonia after lung cancer surgery and the way of measuring both Tumor-SUVmax and NCA-SUVmax. PET, Positron emission tomography; $C T$, computed tomography; DICOM, digital imaging and communications in medicine; 3D, 3-dimensional; SUVmax, maximum standardized uptake value; $I P F$, idiopathic pulmonary fibrosis; NCA, noncancerous lung area; Post-op, postoperative. Video available at: https://www.jtcvs.org/article/S00225223(19)31659-9/fulltext. 


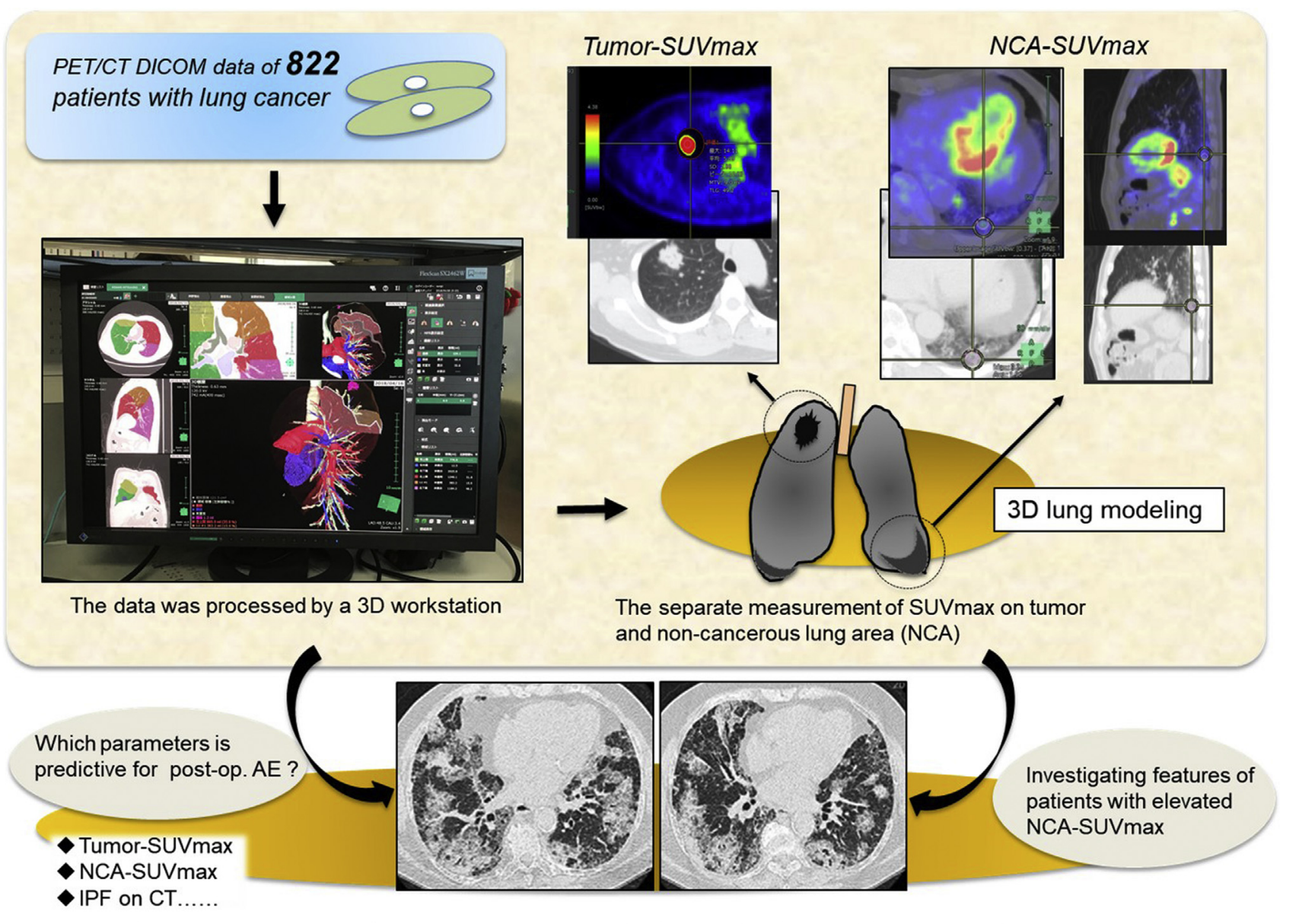

To identify factors associated with acute exacerbation (AE) of interstitial pneumonia after surgery

FIGURE 1. Overview of the current study for the purpose of identifying factors associated with AE of interstitial pneumonia after lung cancer surgery. PET, Positron emission tomography; CT, computed tomography; DICOM, digital imaging and communications in medicine; 3D, 3-dimensional; SUVmax, maximum standardized uptake value; IPF, idiopathic pulmonary fibrosis; NCA, noncancerous lung area; Post-op, postoperative.

indeterminate for UIP and cases without any IP findings) were confirmed by 2 respiratory physicians (S.A. and A.Y.), and a radiologist (R.M.). Patients were excluded if (1) preoperative FDG-PET/CT was not performed; (2) preoperative FDG-PET/CT was performed in other facilities; or (3) the maximum standardized uptake value (SUVmax) was not measurable on a 3-dimensional workstation (Figure 2). Data collection and analyses were approved, and the need to obtain written informed consent from each patient was waived by the Institutional Review Board of Tokyo Medical University.

\section{Definition of Acute Exacerbation and Severe Respiratory Adverse Events}

$\mathrm{AE}$ is defined by the following clinical characteristics: (1) unexplained worsening or development of dyspnea within 30 days; (2) intensified dyspnea; (3) increase in the interstitial shadow on chest radiography and chest CT; (4) decrease in arterial oxygen tension of more than $10 \mathrm{~mm} \mathrm{Hg}$ under similar conditions; (5) no evidence of pulmonary infection; and (6) exclusion of alternative causes, such as cardiac failure, pulmonary embolism, or other identifiable causes of lung injury. ${ }^{17,18}$ SRAEs are defined as grade IIIa or more in the respiratory complications on the Clavian-Dindo classification and represent comprehensive and severe postoperative respiratory adverse events, including AEs, bacterial pneumonia, empyema, and bronchial fistula. ${ }^{16}$

\section{Fluorodeoxyglucose-Positron Emission Tomography/ Computed Tomography Assessment}

All FDG-PET/CT data obtained from the designated facility were used in this study. Patients with blood glucose values of more than $150 \mathrm{mg} / \mathrm{dL}$ during PET/CT image acquisition were excluded. All patients were assessed using a Biograph PET/CT scanner (Siemens Healthcare, Erlangen, Germany). Unenhanced CT images of sections 2 to $4 \mathrm{~mm}$ thick that matched the PET images were acquired from the head to the pelvic floor of each patient using a standard protocol. Immediately after CT, PET covering the identical axial field of view for 2 to 4 minutes per table position depending on the condition of the patient and the scanner performance was conducted. The SUVmax was established by drawing regions of interests (ROIs) around the primary tumor on attenuation-corrected FDG-PET images and calculated using the software integrated within the PET/CT scanner based on the following formula: SUVmax $=\mathrm{C}(\mathrm{MBq} / \mathrm{kg}) /$ $[\operatorname{ID}(\mathrm{MBq}) / \mathrm{w}(\mathrm{kg})]$ in which $\mathrm{C}$ is defined as the maximal activity at a single pixel within the tissue designated by the ROI, and ID is defined as the injected dose/kg of body weight (w). 


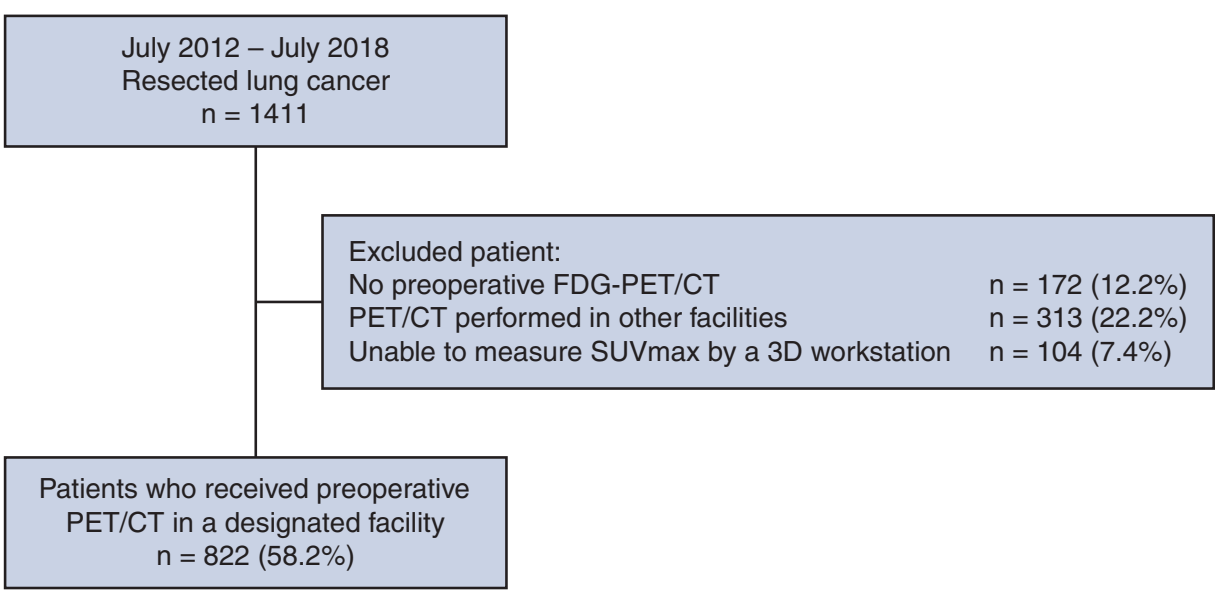

FIGURE 2. Flow chart of the selection of patients analyzed in this study. FDG, Fluorodeoxyglucose; PET, positron emission tomography; $C T$, computed tomography; SUVmax, maximum standardized uptake value; 3D, 3-dimensional.

\section{Measurement of Maximum Standardized Uptake Value of the Main Tumor and Noncancerous Lung Area}

The preoperative FDG-PET/CT images of each patient were transferred to the Synapse Vincent 3D workstation (Fujifilm Corporation, Tokyo, Japan). In every case, both SUVmax of the main tumor (Tumor-SUVmax) and the noncancerous lung area (NCA-SUVmax) were measured. ROIs were drawn, and maximum FDG uptake in the noncancerous lung area was confirmed on HRCT and measured as NCA-SUVmax. ROIs were also measured at least $1 \mathrm{~cm}$ away from the tumor and other organs, such as the ribs, heart, liver, and chest wall. Uptake from the liver and mediastinum was manually excluded. Figure 3 shows representative cases of UIP, probable UIP, and non-UIP.

\section{Statistical Analysis \\ Univariable and multivariable logistic regression analyses were per- formed to identify predictors for AE and SRAEs using the following fac- tors: sex, age, smoking index, tumor size, degree of lymph node dissection (mediastinal vs hilar or none), number of resected lung segments (wedge resection was calculated as 0.5$), \%$ vital capacity ( $\%$ VC), Tumor- SUVmax, NCA-SUVmax, and IPF on CT (yes vs no). A backward step- wise selection method was used to build logistic regression models. The receiver operating characteristic (ROC) area under the curve of significant factors associated with AE and SRAEs was measured, and each cutoff level on the ROC curves for significant parameters associated with $\mathrm{AE}$ and SRAEs represents a sensitivity/specificity pair corresponding to a partic- ular decision threshold. Categoric comparison was performed using the Pearson chi-square test for discrete data and the Mann-Whitney $U$ test or 1-way analysis of variance for continuous data. All tests were 2-sided. The SPSS statistical software package (version 25.0; DDR3 RDIMM, SPSS Inc, Chicago, Ill) was used for statistical analyses.}

\section{RESULTS}

The characteristics of the patients are listed in Table 1. The cohort in this study consisted of 460 men and 362 women (mean age, $67.9 \pm 10.1$ years; range, 23-87 years). Of these, $120(14.6 \%)$ had IPF findings on HRCT. SRAEs were observed in 35 patients $(4.2 \%)$, including those with $\mathrm{AE}(\mathrm{n}=15,1.8 \%)$. The detailed characteristics of patients with AE are summarized in Table E1.
Univariable and multivariable analyses were performed to identify clinico-surgical factors associated with the incidence of AE and SRAEs. Age, sex, \%VC, NCA-SUVmax, smoking index, and IPF on CT were associated with AE on univariable analysis (data not shown), whereas NCASUVmax $(P=.002)$ and the presence of IPF $(P=.002)$ were independent factors on multivariable analysis for $\mathrm{AE}$ (Table 2). Sex, age, tumor size, \%VC, Tumor-SUVmax, NCA-SUVmax, smoking index, the number of resected segments, and the presence of IPF were significantly associated with SRAEs on univariable analysis (data not shown), whereas multivariable analysis demonstrated that age $(P=.022)$, Tumor-SUVmax $(P=.033)$, NCA-SUVmax $(P=.005)$, the number of resected segments $(P=.046)$, and the presence of IPF $(P=.002)$ were independent significant factors for SRAEs (Table 2). We performed univariable and multivariable analyses to investigate factors associated with the incidence of AE and SRAEs, particularly in the 120 patients with IPF. Only NCA-SUVmax was associated with both $\mathrm{AE}$ and SRAEs on univariable analysis (data not shown), whereas NCA-SUVmax was also found to be an independent factor on multivariable analysis for both $\mathrm{AE}(P=.004)$ and SRAEs $(P=.002)$ on multivariable analysis (Table 3).

Quantitative analyses of Tumor-SUVmax and NCASUVmax are shown in Figure 4. In terms of TumorSUVmax, patients with IPF demonstrated higher values than non-IPF patients $(P<.001$; Figure $4, A)$. No statistical difference was observed between patients with UIP and those with probable UIP, whereas values of patients with probable UIP were higher than those of patients with nonUIP $(P<.001$; Figure $4, B)$. Values of patients presenting $\mathrm{AE}$ were higher than those of patients without $\mathrm{AE}$ $(P<.001$; Flgure $4, C)$, whereas values of patients who developed SRAE were higher than those of patients without SRAEs $(P<.001$; Figure $4, D)$. NCA-SUVmax values of 


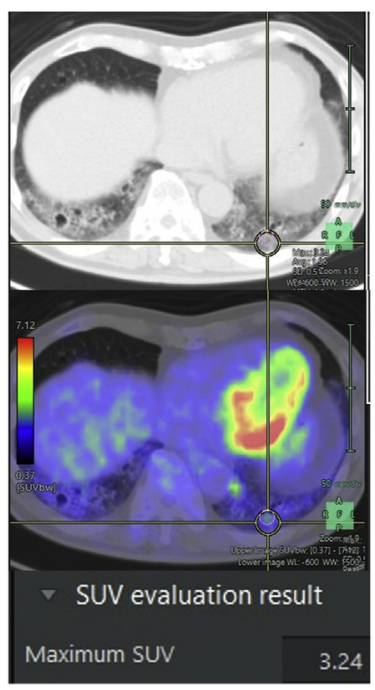

A

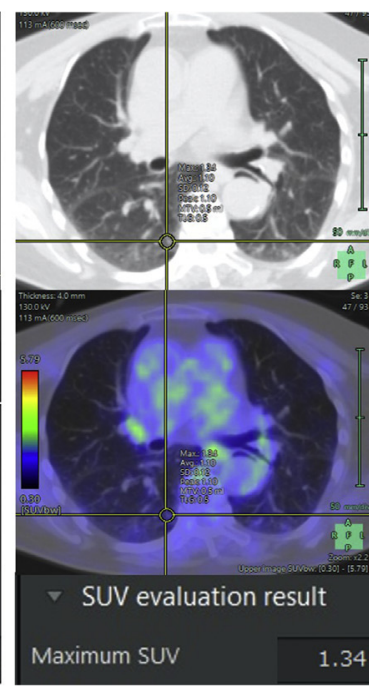

B

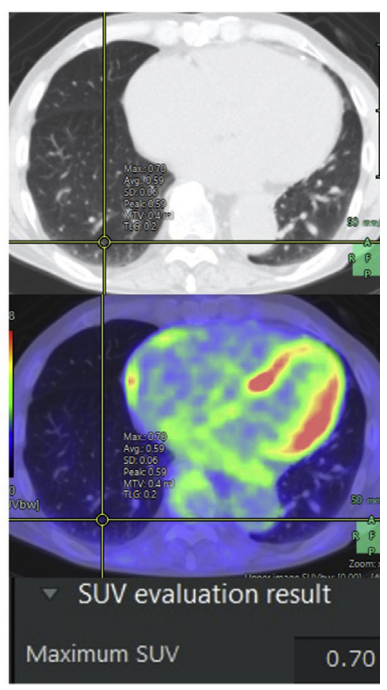

C

FIGURE 3. Representative images of the evaluation results of SUVs in noncancerous lung areas obtained using a 3-dimensional workstation. A, UIP. B, Probable UIP. C, Non-UIP. SUV, Standardized uptake value.

patients with IPF were higher than those of patients with non-IPF $(P<.001$; Figure $4, E)$. Values of patients with UIP were higher than those of patients with probable UIP $(P<.001$; Figure $4, F)$. NCA-SUVmax values of patients who developed $\mathrm{AE}$ were higher than those of patients without AE $(P<.001$; Figure $4, G)$, whereas values of patients who developed SRAE were higher than those of patients without SRAEs $(P<.001$; Figure $4, H)$.

To investigate the effect of NCA-SUVmax on the incidence of AE and SRAEs, we calculated the ROC curves for $\mathrm{AE}$ and SRAEs. The area under the curve and the optimal cutoff value relevant to AE were 0.90 and 1.69, respectively (Figure E1, $A$ ), and those relevant to SRAEs were 0.77 and 1.55 , respectively (Figure E1, B). Table 4 shows a summary of the risk stratification to identify patients presenting $\mathrm{AE}$ and SRAEs according to NCASUVmax and chest CT findings. The list shows that $19.0 \%$ and $30.2 \%$ of patients with positive IPF and NCA-SUV max 1.69 or greater developed AE and SRAEs, respectively.

\section{DISCUSSION}

The 30-day mortality rate after pulmonary resection for patients with lung cancer was reported to be $0.33 \%$ in a Japanese large-scale database. ${ }^{1,19,20} \mathrm{AE}$ is characterized by diffuse and rapid alveolar damage superimposed on a background of IP and is the most frequent cause of death of these patients, accounting for $43.9 \%$ of cases of 30 day mortality. ${ }^{2}$ In the present study, the in-hospital mortality rate for patients with AE of IP after surgery was $60 \%$ (9/ 15 patients). A pretherapeutic risk stratification system for $\mathrm{AE}$ has long been anticipated. Sato and colleagues ${ }^{2}$ reported a predictive score of AE with IP after pulmonary surgery for lung cancer that was established using 7 predictors, namely, history of $\mathrm{AE}$, surgical procedures (wedge resection or more extensive procedures), CT findings, preoperative steroid use, sex, serum KL-6 level, and \% VC, based on a total of 1763 Japanese patients with lung cancer and concomitant interstitial lung disease, and the high-risk group demonstrated that the predicted probability of the incidence of AE was more than $25 \%$. Kumar and colleagues ${ }^{21}$ reported that surgical procedure, lower preoperative diffusion capacity for carbon monoxide $\left(\mathrm{DL}_{\mathrm{CO}}\right)$, and a higher preoperative composite physiologic index calculated with $\mathrm{FEV}_{1.0}$, forced $\mathrm{VC}$, and $\mathrm{DL}_{\mathrm{CO}}$ were associated with postoperative acute respiratory distress syndrome in patients with lung cancer and IPF. Nevertheless, the underlying pathophysiology leading to the acceleration of the onset of $\mathrm{AE}$ remains unclear. Given that there were patients without UIP findings on HRCT who presented with postoperative AE in the current series and published reports, other supplementary or alternative imaging modalities associated with the incidence of $\mathrm{AE}$ are warranted.

F-18 FDG-PET/CT is the standard assessment modality for patients with lung cancer and has a large impact on patient management, initial staging, therapeutic response assessment, and prediction of survival outcomes. ${ }^{10,22}$ Recently, it has been reported that the role of PET/CT extends beyond its clinical use and can be used not only for oncologic assessment but also for evaluation of severity of $\mathrm{IP}^{23}$ In animal studies using a bleomycininduced pulmonary fibrotic murine model, F-18 FDG uptake was associated with the fibrotic process. ${ }^{24,25}$ Bondue and colleagues ${ }^{24}$ reported that F-18 FDG uptake increased 
TABLE 1. Patient background

\begin{tabular}{|c|c|}
\hline Variable & $\mathbf{n}=\mathbf{8 2 2}(\%)$ \\
\hline Age $($ mean $\pm \mathrm{SD})$ & $67.9 \pm 10.1$ \\
\hline \multicolumn{2}{|l|}{ Sex } \\
\hline Men & $460(56.0)$ \\
\hline Women & $362(44.0)$ \\
\hline \multicolumn{2}{|l|}{ Smoking } \\
\hline Yes & $506(61.6)$ \\
\hline No & $316(38.4)$ \\
\hline Brinkmann Index $($ mean \pm SD) & $578.4 \pm 707.4$ \\
\hline \multicolumn{2}{|l|}{$\mathrm{FEV}_{1.0} \%$} \\
\hline$\geq 70$ & $545(66.3)$ \\
\hline$<70$ & $277(33.7)$ \\
\hline \multicolumn{2}{|l|}{$\% \mathrm{VC}$} \\
\hline$\geq 80$ & $804(97.8)$ \\
\hline$<80$ & $18(2.2)$ \\
\hline Tumor-SUVmax (mean $\pm \mathrm{SD})$ & $9.75 \pm 9.20$ \\
\hline IPF on CT & $120(14.6)$ \\
\hline NCA-SUVmax $($ mean \pm SD) & $1.25 \pm 0.53$ \\
\hline \multicolumn{2}{|l|}{ Tumor location } \\
\hline Right upper lobe & $266(32.4)$ \\
\hline Right middle lobe & $51(6.2)$ \\
\hline Right lower lobe & $177(21.5)$ \\
\hline Left upper lobe & $197(24.0)$ \\
\hline Left lower lobe & $131(15.9)$ \\
\hline \multicolumn{2}{|l|}{ Procedure } \\
\hline Wedge resection & $39(4.7)$ \\
\hline Segmentectomy & $45(5.5)$ \\
\hline Lobectomy & $727(88.5)$ \\
\hline Pneumonectomy & $11(1.3)$ \\
\hline \multicolumn{2}{|l|}{ Lymph node dissection } \\
\hline None & $45(5.5)$ \\
\hline Hilar & $94(11.4)$ \\
\hline Mediastinal & $683(83.1)$ \\
\hline \multicolumn{2}{|l|}{ Lung cancer histology } \\
\hline Adenocarcinoma & $641(78.0)$ \\
\hline Squamous cell carcinoma & $108(13.1)$ \\
\hline Others & $73(8.9)$ \\
\hline Duration of surgery, min (mean $\pm \mathrm{SD}$ ) & $211.5 \pm 68.1$ \\
\hline Bleeding, $\mathrm{mL}$ (mean $\pm \mathrm{SD}$ ) & $109.3 \pm 359.4$ \\
\hline \multicolumn{2}{|l|}{ Pathological stage } \\
\hline 0 & $9(1.1)$ \\
\hline I & $545(66.3)$ \\
\hline II & $143(17.4)$ \\
\hline III & $118(14.4)$ \\
\hline IV & $7(0.8)$ \\
\hline \multicolumn{2}{|l|}{ SRAE } \\
\hline $\mathrm{AE}$ & $15(1.8)$ \\
\hline Severe pneumonia & $9(1.1)$ \\
\hline Empyema & $7(0.8)$ \\
\hline Bronchopleural fistula & $4(0.5)$ \\
\hline
\end{tabular}

$S D$, Standard deviation; $F E V$, forced expiratory volume; $V C$, vital capacity; $I P F$, idiopathic pulmonary fibrosis; $C T$, computed tomography; $N C A$, noncancerous lung area; $S U V$, standardized uptake value; $S R A E$, severe respiratory adverse event; $A E$, acute exacerbation.
TABLE 2. Multivariable analysis for factors associated with acute exacerbation and severe respiratory adverse events after surgery $(\mathbf{n}=\mathbf{8 2 2})$

\begin{tabular}{lcc}
\hline \multicolumn{1}{c}{ Variables } & HR $(\mathbf{9 5} \% \mathbf{C I})$ & $\boldsymbol{P}$ value \\
\hline Multivariable analysis for AE & & \\
$\quad$ NCA-SUVmax & $2.301(1.365-3.879)$ & .002 \\
IPF on CT (yes/no) & $8.982(2.543-35.97)$ & .002 \\
Multivariable analysis for SRAEs & & \\
Age & $1.065(1.009-1.124)$ & .022 \\
Tumor-SUVmax & $1.045(1.004-1.089)$ & .033 \\
NCA-SUVmax & $1.926(1.262-2.942)$ & .005 \\
No. of resected segments & $1.258(1.004-1.576)$ & .046 \\
IPF on CT (yes/no) & $3.710(1.592-8.643)$ & .002 \\
\hline
\end{tabular}

$H R$, Hazard ratio; $C I$, confidence interval; $A E$, acute exacerbation; $N C A$, noncancerous lung area; $S U V$, standardized uptake value; $I P F$, idiopathic pulmonary fibrosis; $C T$, computed tomography; $S R A E$, severe respiratory adverse event.

together with the early recruitment of leukocytes during the inflammatory phase and then persisted in the later fibrotic phase in their murine model of lung fibrosis. Recent clinical studies reported that high uptake values of F-18 FDG in the lung parenchyma of patients with IPF were associated with high mortality. ${ }^{13,23}$ In the present study, multivariable analysis of the entire population and the 120 patients with IPF demonstrated that NCA-SUVmax was independently associated with postoperative $\mathrm{AE}$, and the incidences of $\mathrm{AE}$ and SRAEs were $19.0 \%$ and $30.2 \%$, respectively, for patients with IPF and NCA-SUVmax 1.69 or greater. All 12 patients with UIP who developed postoperative AE showed increased NCA-SUVmax values of greater than 1.69, whereas IPF categorization on CT showed a stronger association with NCA-SUVmax than Tumor-SUVmax. These results suggest that lung cancers arising from metabolically active fibrotic parenchyma have the potential of high-grade malignancy, and that these patients need to be considered as being at high risk for life-threatening complications. The integration of CT evaluation of UIP and NCA-SUVmax with PET/CT is more helpful in estimating the risk of postoperative $\mathrm{AE}$ than the individual use of either modality and may enable patients with UIP and increased NCA-SUVmax to receive perioperative

TABLE 3. Multivariable analysis for factors associated with acute exacerbation and severe respiratory adverse events after surgery in patients with idiopathic pulmonary fibrosis $(n=120)$

\begin{tabular}{lcc}
\hline \multicolumn{1}{c}{ Variables } & HR $(\mathbf{9 5} \% \mathbf{C I})$ & $\boldsymbol{P}$ value \\
\hline $\begin{array}{l}\text { Multivariable analysis for AE } \\
\quad \text { NCA-SUVmax }\end{array}$ & $2.952(1.406-6.197)$ & .004 \\
$\begin{array}{l}\text { Multivariable analysis for } \\
\quad \text { SRAEs }\end{array}$ & & \\
$\quad$ NCA-SUVmax & $2.643(1.335-5.234)$ & .002 \\
\hline
\end{tabular}

$H R$, Hazard ratio; $C I$, confidence interval; $A E$, acute exacerbation; $N C A$, noncancerous lung area; $S U V$, standardized uptake value; $S R A E$, severe respiratory adverse event. 


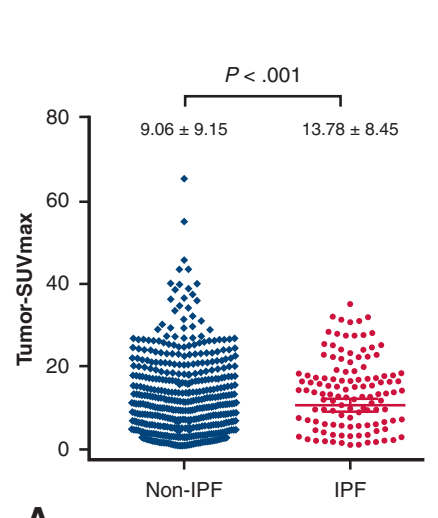

A
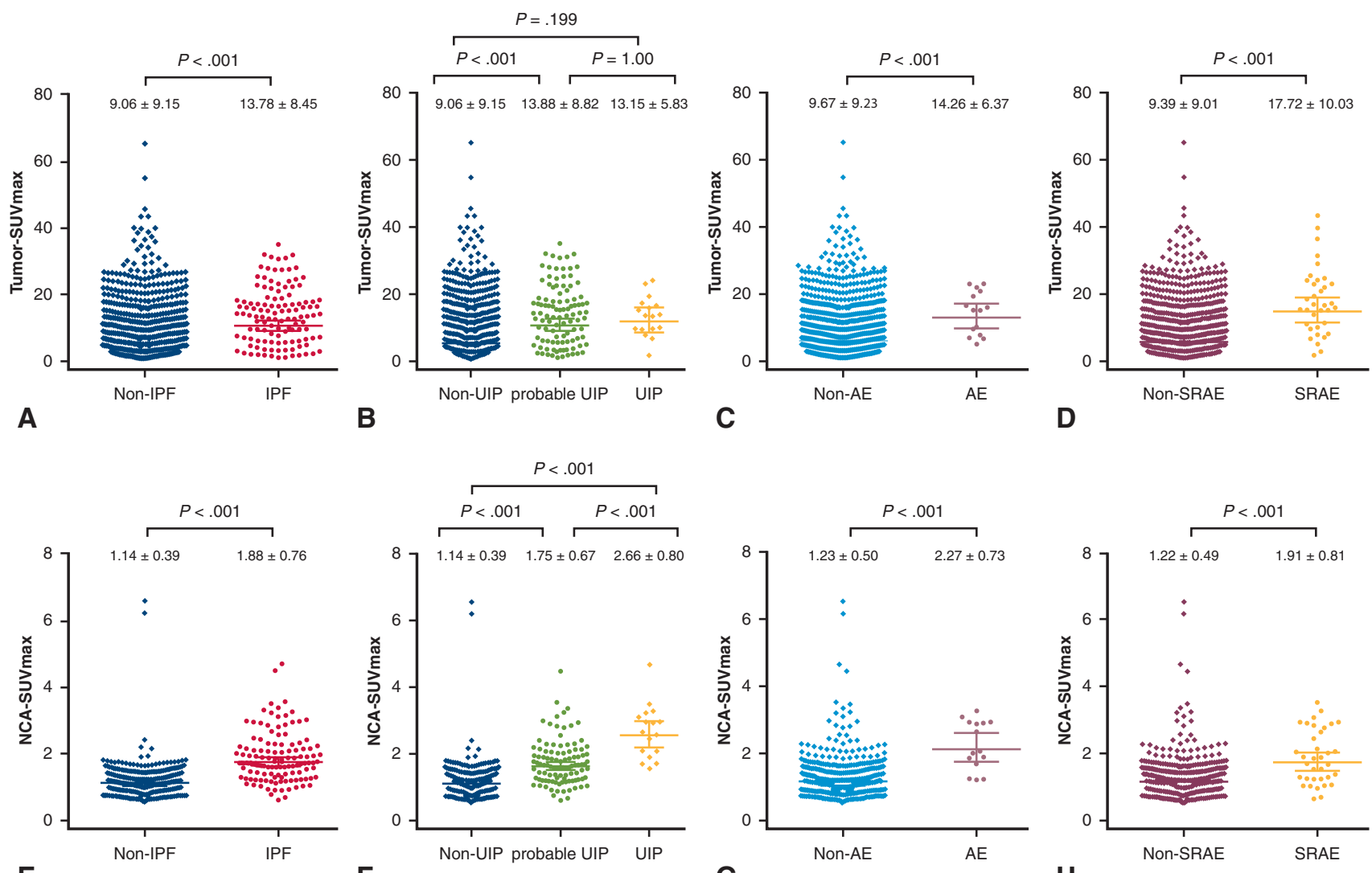

C

D
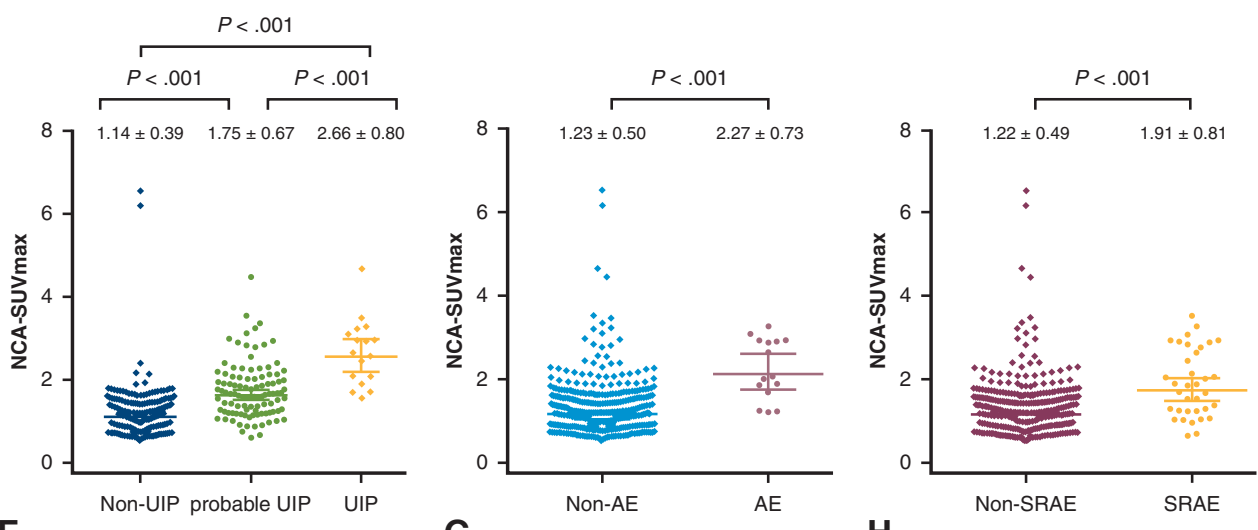

FIGURE 4. Comparison of maximum standardized uptake values between lung tumors (Tumor-SUVmax) and noncancerous lung areas (NCA-SUVmax). A, Patients with IPF demonstrated significantly higher Tumor-SUVmax values than those in patients without IPF. B, There was a significant difference in Tumor-SUVmax values between patients with probable UIP and those without UIP, but no difference between patients with UIP and those with probable UIP. C, Tumor-SUVmax values of patients presenting AE was higher than those of patients without AE. D, Tumor-SUVmax values of patients presenting SRAEs were higher than those of patients without SRAEs. E, NCA-SUVmax values of patients with IPF were higher than those of patients without IPF. F, NCA-SUVmax values in patients with UIP were significantly higher than those in patients with probable UIP. G, NCA-SUVmax values of patients presenting AE were higher than those of patients without AE. H, NCA-SUVmax values of patients presenting SRAE were higher than those of patients without SRAEs. IPF, Idiopathic pulmonary fibrosis; UIP, usual interstitial pneumonitis; $A E$, acute exacerbation; SRAE, severe respiratory adverse event.

prophylactic treatments, such as corticosteroids, sivelestat, nintedanib, and pirfenidone, or alternative treatments according to the comorbidity status of the patient. Recently, much attention has been focused on pretherapeutic identification for immune therapy-induced lung injury and radiation-induced pneumonitis before its physiologic manifestation. Of the 3 patients with IPF who underwent radiotherapy in our hospital in 2018, 2 patients with increased NCA-SUVmax values of greater than 2 developed AE (data not shown). On the other hand, the 2

TABLE 4. Risk stratification to identify acute exacerbations and severe respiratory adverse events after surgery

\begin{tabular}{lccccc}
\hline \multicolumn{1}{c}{ Variables } & No. of patients & No. of AEs & Proportion of AEs (\%) & No. of SRAEs & Proportion of SRAEs (\%) \\
\hline All patients & 822 & 15 & 1.8 & 35 & 4.3 \\
Patients with IPF & 120 & 12 & 10.0 & 21 & 17.5 \\
\hline Patient with NCA-SUVmax $\geq 1.55$ & 129 & 12 & 9.3 & 22 & 17.1 \\
Patient with NCA-SUVmax $\geq 1.69$ & 83 & 12 & 14.5 & 19 & 22.9 \\
Patients with IPF + NCA-SUV max $\geq 1.55$ & 80 & 12 & 15.0 & 20 & 25.0 \\
Patients with IPF + NCA-SUV max $\geq 1.69$ & 63 & 12 & 19.0 & 19 & 30.2 \\
\hline
\end{tabular}

$A E$, Acute exacerbation; $S R A E$, severe respiratory adverse events; $I P F$, idiopathic pulmonary fibrosis; $N C A$, noncancerous lung area; $S U V$, standardized uptake value. 
patients with NCA-SUVmax values below the cutoff who received programmed death-1 inhibitors did not develop $\mathrm{AE}$ (data not shown). The importance of measuring Tumor-SUVmax and NCA-SUVmax separately needs to be confirmed by a larger prospective study for the potential practical use of these parameters.

\section{Study Limitations}

This study has several limitations. First, the optimal cutoff value of SUVmax that dichotomized the 2 groups is only valid in the present population because of its variability by the number of patients, plasma glucose concentration, and type of scanner. To partly overcome this problem, an anthropomorphic body phantom study to adjust quantitative SUVs should be performed when a multi-institutional observational study is performed. Second, whereas AE-IPF is clinically important, its rareness makes it difficult to study in a large number of patients, causing inadequate statistical power that might not ensure a realistic chance of obtaining significant results. It is also occasionally difficult to accurately diagnose this condition from CT findings in the presence of an AE. Third, the diagnostic approach for IPF in this study is based only on HRCT features. We did not always perform lung cancer surgery together with surgical lung biopsy of different lobes with the intention to diagnosing IPF.

\section{CONCLUSIONS}

NCA-SUVmax was independently associated with the incidence of $\mathrm{AE}$ and SRAEs on multivariable analysis in the entire population and in patients with IPF. FDG-PET/ CT imaging of patients with lung cancer contributes to daily clinical practice not only for the assessment of malignant tumor grade but also for the identification of individuals at high risk of postoperative $\mathrm{AE}$ and will assist in complex clinical decision-making.

\section{Conflict of Interest Statement}

Authors have nothing to disclose with regard to commercial support.

The authors thank the medical editors from the Department of International Medical Communications of Tokyo Medical University for editing the English manuscript. And Mami Murakami for assistance with the statistical analyses of this work.

\section{References}

1. Watanabe A, Kawaharada N, Higami T. Postoperative acute exacerbation of IPF after lung resection for primary lung cancer. Pulm Med. 2011;2011:960316.

2. Sato T, Teramukai S, Kondo H, Watanabe A, Ebina M, Kishi K, et al. Impact and predictors of acute exacerbation of interstitial lung diseases after pulmonary resection for lung cancer. J Thorac Cardiovasc Surg. 2014;147:1604-11.e1603.

3. Sato T, Kondo H, Watanabe A, Nakajima J, Niwa H, Horio H, et al. A simple risk scoring system for predicting acute exacerbation of interstitial pneumonia after pulmonary resection in lung cancer patients. Gen Thorac Cardiovasc Surg. 2015;63:164-72.
4. Zank DC, Bueno M, Mora AL, Rojas M. Idiopathic pulmonary fibrosis: aging, mitochondrial dysfunction, and cellular bioenergetics. Front Med (Lausanne). 2018;5:10.

5. Xie N, Tan Z, Banerjee S, Cui H, Ge J, Liu RM, et al. Glycolytic reprogramming in myofibroblast differentiation and lung fibrosis. Am J Respir Crit Care Med. 2015;192:1462-74.

6. Maher TM. Aerobic glycolysis and the Warburg effect. An unexplored realm in the search for fibrosis therapies? Am J Respir Crit Care Med. 2015;192:1407-9.

7. Pardo A, Selman M. Lung fibroblasts, aging, and idiopathic pulmonary fibrosis. Ann Am Thorac Soc. 2016;13(Suppl 5):S417-21.

8. Choi WH, Yoo IR, O JH, Kim TJ, Lee KY, Kim YK. Is the Glut expression related to FDG uptake in PET/CT of non-small cell lung cancer patients? Technol Health Care. 2015;23(Suppl 2):S311-8.

9. Uehara H, Tsutani Y, Okumura S, Nakayama H, Adachi S, Yoshimura M, et al. Prognostic role of positron emission tomography and high-resolution computed tomography in clinical stage IA lung adenocarcinoma. Ann Thorac Surg. 2013; 96:1958-65.

10. Kaira K, Serizawa M, Koh Y, Takahashi T, Yamaguchi A, Hanaoka H, et al. Biological significance of 18F-FDG uptake on PET in patients with non-small-cell lung cancer. Lung Cancer. 2014;83:197-204.

11. Nobashi T, Kubo T, Nakamoto Y, Handa T, Koyasu S, Ishimori T, et al. 18F-FDG uptake in less affected lung field provides prognostic stratification in patients with interstitial lung disease. J Nucl Med. 2016;57:1899-904.

12. Groves AM, Win T, Screaton NJ, Berovic M, Endozo R, Booze H, et al. Idiopathic pulmonary fibrosis and diffuse parenchymal lung disease: implications from initial experience with 18F-FDG PET/CT. J Nucl Med. 2009;50: $538-45$.

13. Flechsig P, Hural O, Kreuter M, Eichhorn M, HEUßEL G, Sachpekidis C, et al. Impact of FDG-PET on the detection of patients with lung cancer at high risk for ILD. In Vivo. 2018;32:1457-62.

14. McDonough JE, Martens DS, Tanabe N, Ahangari F, Verleden SE, Maes K, et al. A role for telomere length and chromosomal damage in idiopathic pulmonary fibrosis. Respir Res. 2018;19:132.

15. Kim SJ, Cheresh P, Jablonski RP, Williams DB, Kamp DW. The role of mitochondrial DNA in mediating alveolar epithelial cell apoptosis and pulmonary fibrosis. Int J Mol Sci. 2015;16:21486-519.

16. Dindo D, Demartines N, Clavien PA. Classification of surgical complications: a new proposal with evaluation in a cohort of 6336 patients and results of a survey. Ann Surg. 2004;240:205-13.

17. Raghu G, Collard HR, Egan JJ, Martinez FJ, Behr J, Brown KK, et al. An official ATS/ERS/JRS/ALAT statement: idiopathic pulmonary fibrosis: evidence-based guidelines for diagnosis and management. Am J Respir Crit Care Med. 2011; 183:788-824.

18. Yoshimura K, Nakatani T, Nakamori Y, Chonabayashi N, Tachibana A, Nakata K, et al. [Acute exacerbation in idiopathic interstitial pneumonia]. Nihon Kyobu Shikkan Gakkai Zasshi. 1984;22:1012-20.

19. Okumura M. Trends and current status of general thoracic surgery in Japan revealed by review of nationwide databases. J Thorac Dis. 2016;8:S589-95.

20. Raghu G, Nyberg F, Morgan G. The epidemiology of interstitial lung disease and its association with lung cancer. Br J Cancer. 2004;91(Suppl 2):S3-10.

21. Kumar P, Goldstraw P, Yamada K, Nicholson AG, Wells AU, Hansell DM, et al. Pulmonary fibrosis and lung cancer: risk and benefit analysis of pulmonary resection. J Thorac Cardiovasc Surg. 2003;125:1321-7.

22. Furumoto H, Shimada Y, Imai K, Maehara S, Maeda J, Hagiwara M, et al. Prognostic impact of the integration of volumetric quantification of the solid part of the tumor on 3DCT and FDG-PET imaging in clinical stage IA adenocarcinoma of the lung. Lung Cancer. 2018;121:91-6.

23. Umeda Y, Demura Y, Morikawa M, Anzai M, Kadowaki M, Ameshima S, et al. Prognostic value of dual-time-point 18F-FDG PET for idiopathic pulmonary fibrosis. J Nucl Med. 2015;56:1869-75.

24. Bondue B, Sherer F, Van Simaeys G, Doumont G, Egrise D, Yakoub Y, et al. PET/ CT with 18F-FDG- and 18F-FBEM-labeled leukocytes for metabolic activity and leukocyte recruitment monitoring in a mouse model of pulmonary fibrosis. $\mathrm{J} \mathrm{Nucl}$ Med. 2015;56:127-32.

25. Bondue B, Castiaux A, Van Simaeys G, Mathey C, Sherer F, Egrise D, et al. Absence of early metabolic response assessed by 18F-FDG PET/CT after initiation of antifibrotic drugs in IPF patients. Respir Res. 2019;20:10.

Key Words: acute exacerbation, idiopathic pulmonary fibrosis, lung cancer, SUVmax 


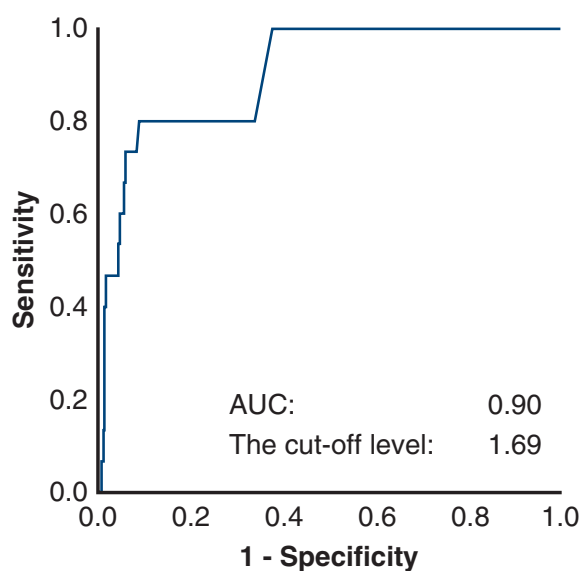

A

FIGURE E1. The receiver operating characteristic areas under the curves for maximum standardized uptake values (SUVmax) in noncancerous lung area used to identify the probability of AE (A) and severe respiratory adverse events (B) after surgery. AUC, Area under the curve.

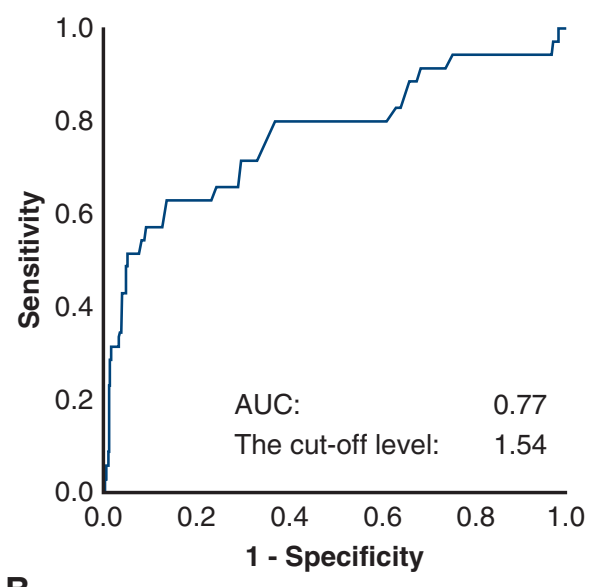

B 
TABLE E1. Background of patients with acute exacerbation of idiopathic pulmonary fibrosis

\begin{tabular}{|c|c|c|c|c|c|c|c|c|c|c|c|c|c|}
\hline Patient & Sex & Age $(y)$ & $\begin{array}{c}\text { Administration } \\
\text { of preoperative } \\
\text { PSL }\end{array}$ & $\begin{array}{c}\text { Classification } \\
\text { of IP }\end{array}$ & $\begin{array}{c}\text { KL-6 } \\
\text { level } \\
(\mathrm{U} / \mathbf{m L})\end{array}$ & $\begin{array}{l}\text { NCA-SUV } \\
\max \end{array}$ & Tumor-SUVmax & $\begin{array}{c}\% \mathrm{VC} \\
(\%)\end{array}$ & $\begin{array}{c}\text { FEV }_{1.0} \\
(\%)\end{array}$ & $\begin{array}{l}\text { Surgical procedure (degree } \\
\text { of lymph node dissection) }\end{array}$ & Histology & $\begin{array}{l}\text { Pathological } \\
\text { stage }\end{array}$ & Outcome* \\
\hline 1 & $\mathrm{~F}$ & 81 & No & Probable UIP & - & 2.07 & 4.80 & 108.1 & 75.2 & Lobectomy (Mediastinal) & Adenocarcinoma & IIA & Alive \\
\hline 2 & M & 65 & No & UIP & 4783 & 2.95 & 19.1 & 83.4 & 81.3 & Wedge resection (None) & Adenocarcinoma & IB & Alive \\
\hline 3 & M & 79 & No & Probable UIP & 929 & 2.90 & 21.8 & 108.2 & 60.0 & Lobectomy (Hilar) & Squamous & IB & Deceased \\
\hline 4 & M & 72 & Yes & Probable UIP & - & 2.94 & 22.8 & 113.3 & 76.8 & Lobectomy (Mediastinal) & Adenocarcinoma & IIIA & Alive \\
\hline 5 & M & 71 & No & Non-UIP & - & 1.25 & 16.3 & 109.5 & 60.9 & $\begin{array}{l}\text { Pneumonectomy } \\
\text { (Mediastinal) }\end{array}$ & Squamous & IIIB & Deceased \\
\hline 6 & M & 78 & No & UIP & 965 & 3.09 & 22.8 & 87.9 & 70.7 & Lobectomy (Mediastinal) & Pleomorphic & IIB & Deceased \\
\hline 7 & M & 66 & No & UIP & 351 & 1.90 & 9.40 & 97.7 & 43.0 & Wedge resection (None) & Adenocarcinoma & IIA & Alive \\
\hline 8 & M & 73 & No & UIP & 1281 & 3.28 & 15.7 & 60.1 & 93.0 & Lobectomy (Mediastinal) & Squamous & IIIA & Deceased \\
\hline 9 & M & 60 & No & UIP & 4636 & 2.65 & 15.1 & 97.3 & 79.1 & Lobectomy (Hilar) & Squamous & IA & Deceased \\
\hline 10 & M & 76 & No & Probable UIP & - & 1.86 & 15.0 & 127.6 & 74.6 & Lobectomy (Mediastinal) & Squamous & IIA & Deceased \\
\hline 11 & M & 73 & No & Non-UIP & - & 1.24 & 20.7 & 109.9 & 78.0 & Lobectomy (Mediastinal) & Pleomorphic & IIA & Deceased \\
\hline 12 & M & 79 & No & UIP & 681 & 1.70 & 7.56 & 65.1 & 73.6 & Lobectomy (Mediastinal) & Squamous & IIA & Deceased \\
\hline 13 & M & 74 & Yes & UIP & 934 & 2.92 & 6.45 & 84.4 & 69.0 & Lobectomy (Mediastinal) & SCLC & IIIA & Alive \\
\hline 14 & M & 74 & No & Non-UIP & - & 1.23 & 9.87 & 100 & 76.9 & Lobectomy (Mediastinal) & Adenocarcinoma & IB & Alive \\
\hline 15 & M & 78 & No & Probable UIP & 612 & 2.02 & 6.79 & 110.7 & 72.0 & Lobectomy (Mediastinal) & Adenosquamous & IB & Deceased \\
\hline
\end{tabular}

$\overline{P S L}$, Prednisolone; $I P$, interstitial pneumonia; $K L-6$, sialylated carbohydrate antigen Krebs von den Lungen-6; $N C A$, noncancerous lung area; $S U V m a x$, maximum standardized uptake value; $V C$, vital capacity; $F E V$, forced expiratory volume; UIP, usual interstitial pneumonitis; $S C L C$, small cell lung cancer. *Cause of death described as due to acute exacerbation. 